\title{
Trace formulae for Schrödinger operators with complex-valued potentials on cubic lattices
}

\author{
Evgeny Korotyaev ${ }^{1} \cdot$ Ari Laptev $^{2}$
}

Received: 4 January 2018 / Accepted: 22 January 2018 / Published online: 13 March 2018

(C) The Author(s) 2018. This article is an open access publication

\begin{abstract}
We consider a class of Schrödinger operators with complex decaying potentials on the lattice. Using some classical results from complex analysis we obtain some trace formulae and use them to estimate zeros of the Fredholm determinant in terms of the potential.
\end{abstract}

Keywords Scattering $\cdot$ Trace formula $\cdot$ Lattice $\cdot$ Complex eigenvalues $\cdot$ Hardy spaces

Mathematics Subject Classification 34A55 · (34B24, 47E05)

\section{Introduction}

Let us consider a Schrödinger operator $H$ acting in $\ell^{2}\left(\mathbb{Z}^{d}\right), d \geqslant 3$, given by

$$
H=H_{0}+V, \quad H_{0}=\Delta,
$$

where $\Delta$ is the discrete Laplacian on $\mathbb{Z}^{d}$ defined by

$$
(\Delta f)(n)=\frac{1}{2} \sum_{j=1}^{d}\left(f\left(n+e_{j}\right)+f\left(n-e_{j}\right)\right), \quad n=\left(n_{j}\right)_{j=1}^{d} \in \mathbb{Z}^{d},
$$

Communicated by Efim Zelmanov.

$\triangle$ Ari Laptev

a.laptev@imperial.ac.uk

Evgeny Korotyaev

korotyaev@gmail.com; e.korotyaev@spbu.ru

1 Saint-Petersburg State University, Universitetskaya nab. 7/9, St. Petersburg, Russia 199034

2 Imperial College London, London, UK 
for $f=\{f(n)\}_{n \in \mathbb{Z}^{d}} \in \ell^{2}\left(\mathbb{Z}^{d}\right)$. Here $e_{1}=(1,0, \ldots, 0), \ldots, e_{d}=(0, \ldots, 0,1)$ is the standard basis of $\mathbb{Z}^{d}$. The operator $V=\{V(n)\}_{n \in \mathbb{Z}^{d}}, V(n) \in \mathbb{C}$, is a complex potential defined by

$$
(V f)(n)=V(n) f(n), \quad n \in \mathbb{Z}^{d} .
$$

We assume that the potential $V$ satisfies the following condition:

$$
V \in \ell^{2 / 3}\left(\mathbb{Z}^{d}\right) \text {. }
$$

The condition (1.1) implies that $V$ can be factorised as

$$
V=V_{1} V_{2}, \quad \text { where } V_{1} \in \ell^{1}\left(\mathbb{Z}^{d}\right), V_{2} \in \ell^{2}\left(\mathbb{Z}^{d}\right),
$$

with $V_{1}=|V|^{2 / 3-1} V$ and $V_{2}=|V|^{1 / 3}$.

Here $\ell^{q}\left(\mathbb{Z}^{d}\right), q>0$, is the space of sequences $f=\{f(n)\}_{n \in \mathbb{Z}^{d}}$ such that $\|f\|_{q}<\infty$, where

$$
\|f\|_{q}=\|f\|_{\ell q\left(\mathbb{Z}^{d}\right)}= \begin{cases}\sup _{n \in \mathbb{Z}^{d}}|f(n)|, & q=\infty, \\ \left(\sum_{n \in \mathbb{Z}^{d}}|f(n)|^{q}\right)^{\frac{1}{q}}, & q \in(0, \infty) .\end{cases}
$$

If $q \geqslant 1$ then $\ell^{q}\left(\mathbb{Z}^{d}\right)$ is the Banach space equipped with the norm $\|\cdot\|_{q}$. It is well-known that the spectrum of the Laplacian $\Delta$ is absolutely continuous and equals

$$
\sigma(\Delta)=\sigma_{\mathrm{ac}}(\Delta)=[-d, d] .
$$

It is also known that if $V$ satisfies (1.1), then the essential spectrum of the Schrödinger operator $H$ on $\ell^{2}\left(\mathbb{Z}^{d}\right)$ is

$$
\sigma_{\mathrm{ess}}(H)=[-d, d]
$$

However, this condition does not exclude appearance of the singular continuous spectrum on the interval $[-d, d]$. Our main goal is to find new trace formulae for the operator $H$ with complex potentials $V$ and to use these formulae for some estimates of complex eigenvalues in terms of the potential function.

Some of the results obtained in this paper are new even in the case of real-valued potentials due to the presence of the measure $\sigma$ (see Theorem 2.3) appearing in the canonical factorisation of the respective Fredholm determinants. Non-triviality of such a measure is due to the condition (1.1) on the potential $V$. We believe that it would be interesting to study the deeper relation between properties of $V$ and the measure $\sigma$.

Recently uniform bounds on eigenvalues of Schrödinger operators in $\mathbb{R}^{d}$ with complex-valued potentials decaying at infinity attracted attention of many specialists. We refer to [6] for a review of the state of the art of non-selfadjoint Schrödinger operators and for motivations and applications. Bounds on single eigenvalues were proved, for instance, in $[1,7,11,16]$ and bounds on sums of powers of eigenvalues were found in $[4,8,9,12,13,15,17,26]$. The latter bounds generalise the Lieb-Thirring 
bounds [27] to the non-selfadjoint setting. In [15] (Theorem 16) Frank and Sabin have obtained estimates on the sum of the distances between the complex eigenvalues and the continuous spectrum $[0, \infty)$ in terms of $L^{p}$-norms of the potentials. However, almost no results are known on the number of eigenvalues of Schrödinger operators with complex potentials. We referee here to the paper [14] where the authors discussed this problem in details in odd dimensions.

In [34] Safronov has obtained some trace formulae in the continuous setting showing that the series of imaginary parts of square roots of eigenvalues is convergent. Besides, he proved that if $|V|$ decays faster than the Coulomb potential, then all eigenvalues are inside a disc of a finite radius.

For the discrete Schrödinger operators most of the results were obtained in the self-adjoint setting, see, for example, [37] where the operator on $\mathbb{Z}^{1}$ were studied. Schrödinger operators with decreasing potentials on the lattice $\mathbb{Z}^{d}$ have been considered by Boutet de Monvel-Sahbani [5], Isozaki-Korotyaev [21], Kopylova [24], Rosenblum-Solomjak [30], Shaban-Vainberg [35] and see references therein. Ando [2] studied the inverse spectral theory for the discrete Schrödinger operators with finitely supported potentials on the hexagonal lattice. Scattering on periodic metric graphs $\mathbb{Z}^{d}$ were considered by Korotyaev-Saburova [25]. Isozaki and Morioka (see Theorem 2.1 in [22]) proved that if the potential $V$ is real and compactly supported, then the point-spectrum of $H$ on the interval $(-d, d)$ is absent. Note that in [22] the authors gave an example of embedded eigenvalue at the end points $\{ \pm d\}$.

In this paper we use classical results from complex analysis that give us a new class of trace formulae for the spectrum of discrete multi-dimensional Scrödinger operators with complex-valued potentials. In particular, we consider a so-called canonical factorisation of analytic functions from Hardy spaced via its inner and outer factors, see Sect. 6. Such factorisations applied to Fredholm determinants allow us to obtain trace formula that lead to some inequalities on the complex spectrum in terms of the $L^{2 / 3}$ norm of the potential function. Note also that in the case $d=3$ we use a delicate uniform inequality for Bessel's functions obtained in Lemma 7.1.

\section{Some notations and statements of main results}

We denote by $\mathbb{D}_{r}\left(z_{0}\right) \subset \mathbb{C}$ the disc with radius $r>0$ and center $z_{0} \in \mathbb{C}$

$$
\mathbb{D}_{r}\left(z_{0}\right)=\left\{z \in \mathbb{C}:\left|z-z_{0}\right|<r\right\},
$$

and abbreviate $\mathbb{D}_{r}=\mathbb{D}_{r}(0)$ and $\mathbb{D}=\mathbb{D}_{1}$. Let also $\mathbb{T}=\mathbb{R} /(2 \pi \mathbb{Z})$. It is convenient to introduce a new spectral variable $z \in \mathbb{D}$ by

$$
\lambda=\lambda(z)=\frac{d}{2}\left(z+\frac{1}{z}\right) \in \Lambda=\mathbb{C} \backslash[-d, d], \quad z \in \mathbb{D} .
$$

The function $\lambda(z)$ has the following properties:

- $z \rightarrow \lambda(z)$ is a conformal mapping from $\mathbb{D}$ onto the spectral domain $\Lambda$.

- $\lambda(\mathbb{D})=\Lambda=\mathbb{C} \backslash[-d, d]$ and $\lambda\left(\mathbb{D} \cap \mathbb{C}_{\mp}\right)=\mathbb{C}_{ \pm}$. 
- $\Lambda$ is the cut domain with the cut $[-d, d]$, having the upper side $[-d, d]+i 0$ and the lower side $[-d, d]-i 0 . \lambda(z)$ maps the boundary: the upper semi-circle onto the lower side $[-d, d]-i 0$ and the lower semi-circle onto the upper side $[-d, d]+i 0$.

- $\lambda(z)$ maps $z=0$ to $\lambda=\infty$.

- The inverse mapping $z(\cdot): \Lambda \rightarrow \mathbb{D}$ is given by

$$
\begin{aligned}
& z=\frac{1}{d}\left(\lambda-\sqrt{\lambda^{2}-d^{2}}\right), \quad \lambda \in \Lambda, \\
& z=\frac{d}{2 \lambda}+\frac{O(1)}{\lambda^{3}}, \quad \text { as } \quad|\lambda| \rightarrow \infty .
\end{aligned}
$$

Assume that $F$ is analytic in $\mathbb{D}$. We say that $F$ belongs the Hardy space $\mathscr{H}_{\infty}$ if

$$
\|F\|_{\mathscr{H}_{\infty}}=\sup _{z \in \mathbb{D}}|F(z)| .
$$

Let $\mathcal{B}$ denote the class of bounded operators and $\mathcal{B}_{1}$ and $\mathcal{B}_{2}$ be the trace and the Hilbert-Schmidt class equipped with the norm $\|\cdot\|_{\mathcal{B}_{1}}$ and $\|\cdot\|_{\mathcal{B}_{2}}$ respectively.

Denote by $D(z), z \in \mathbb{D}$, the determinant

$$
D(z)=\operatorname{det}\left(I+V R_{0}(\lambda(z)), \quad z \in \mathbb{D},\right.
$$

where $R_{0}(\lambda)=\left(H_{0}-\lambda\right)^{-1}, \lambda \in \Lambda$. The determinant $D(z), z \in \mathbb{D}$, is well defined for $V \in \mathcal{B}_{1}$ and if $\lambda_{0} \in \Lambda$ is an eigenvalue of $H$, then $z_{0}=z\left(\lambda_{0}\right) \in \mathbb{D}$ is a zero of $D$ with the same algebraic multiplicity. Below we consider only algebraic multiplicity of eigenvalues.

Theorem 2.1 Let a potential $V$ satisfy the condition (1.1). Then the determinant $D(z)=\operatorname{det}\left(I+V R_{0}(\lambda(z)), z \in \mathbb{D}\right.$, is analytic in $\mathbb{D}$ and Hölder up to the boundary. It has $N \leqslant \infty$ zeros $\left\{z_{j}\right\}_{j=1}^{N}$, such that

$$
\begin{aligned}
& 0<r_{0}=\left|z_{1}\right| \leqslant\left|z_{2}\right| \leqslant \cdots \leqslant\left|z_{j}\right| \leqslant\left|z_{j+1} \leqslant\right| z_{j+2} \mid \leqslant \cdots, \\
& \text { where } r_{0}=\inf \left|z_{j}\right|>0
\end{aligned}
$$

Moreover, it satisfies

$$
\|D\|_{\mathscr{H}_{\infty}} \leqslant e^{C\|V\|_{2 / 3}},
$$

where the constant $C$ depends only on $d$. Furthermore, the function $\log D(z)$ whose branch is defined by $\log D(0)=0$, is analytic in the disk $\mathbb{D}_{r_{0}}$ with the radius $r_{0}>0$ defined by (2.2) and it has the Taylor series as $|z|<r_{0}$ :

$$
\log D(z)=-c_{1} z-c_{2} z^{2}-c_{3} z^{3}-c_{4} z^{4}-\cdots
$$

where

$$
\begin{aligned}
& c_{1}=d_{1} a, \quad c_{2}=d_{2} a^{2}, \quad c_{3}=d_{3} a^{3}-c_{1}, \quad c_{4}=d_{4} a^{4}-c_{2}, \ldots, \\
& d_{1}=\operatorname{Tr} V, \quad d_{2}=\operatorname{Tr} V^{2}, \quad d_{3}=\operatorname{Tr}\left(V^{3}+(3 d / 2) V\right), \ldots,
\end{aligned}
$$


and where $a=\frac{2}{d}$.

Define the Blaschke product $B(z), z \in \mathbb{D}$ by

$$
\begin{array}{ll}
B(z)=\prod_{j=1}^{N} \frac{\left|z_{j}\right|}{z_{j}} \frac{\left(z_{j}-z\right)}{\left(1-\bar{z}_{j} z\right)}, & \text { if } \quad N \geqslant 1, \\
B=1, & \text { if } \quad N=0 .
\end{array}
$$

Theorem 2.2 Let a potential $V$ satisfy the condition (1.1) and let $N \geqslant 1$. Then zeros $\left\{z_{j}\right\}$ of $D$ in the disk $\mathbb{D}$ labeled by (2.2) satisfy

$$
\sum_{j=1}^{N}\left(1-\left|z_{j}\right|\right)<\infty .
$$

Moreover, the Blaschke product $B(z), z \in \mathbb{D}$, given by (2.7) converges absolutely for $\{|z|<1\}$ and satisfies

(i) $B \in \mathscr{H}_{\infty}$ with $\|B\|_{\mathscr{H}_{\infty}} \leqslant 1$,

$$
\lim _{r \rightarrow 1}\left|B\left(r e^{i \vartheta}\right)\right|=\left|B\left(e^{i \vartheta}\right)\right|=1 \text { for almost all } \vartheta \in \mathbb{T},
$$

and

$$
\lim _{r \rightarrow 1} \int_{0}^{2 \pi} \log \left|B\left(r e^{i \vartheta}\right)\right| d \vartheta=0 .
$$

(ii) The determinant $D$ has the factorization in the disc $\mathbb{D}$ :

$$
D=B D_{B}
$$

where $D_{B}$ is analytic in the unit disc $\mathbb{D}$ and has no zeros in $\mathbb{D}$.

(iii) The Blaschke product $B$ has Taylor's expansion at $z=0$ :

$$
\log B(z)=B_{0}-B_{1} z-B_{2} z^{2}-\cdots, \text { as } z \rightarrow 0,
$$

where $B_{n}$ satisfy

$$
\begin{aligned}
B_{0} & =\log B(0)<0, \quad B_{1}=\sum_{j=1}^{N}\left(\frac{1}{z_{j}}-\bar{z}_{j}\right), \ldots, \quad B_{n}=\frac{1}{n} \sum_{j=1}^{N}\left(\frac{1}{z_{j}^{n}}-\bar{z}_{j}^{n}\right) \ldots, \\
\left|B_{n}\right| & \leqslant \frac{2}{r_{0}^{n}} \sum_{j=1}^{N}\left(1-\left|z_{j}\right|\right) .
\end{aligned}
$$

The next statement describes the canonical representation of the determinant $D(z)$. 
Theorem 2.3 Let a potential $V$ satisfy the condition (1.1). Then

(i) There exists a singular measure $\sigma \geqslant 0$ on $[-\pi, \pi]$, such that the determinant $D$ has a canonical factorization for all $|z|<1$ given by

$$
\begin{aligned}
& D(z)=B(z) e^{-K_{\sigma}(z)} e^{K_{D}(z)}, \\
& K_{\sigma}(z)=\frac{1}{2 \pi} \int_{-\pi}^{\pi} \frac{e^{i t}+z}{e^{i t}-z} d \sigma(t), \\
& K_{D}(z)=\frac{1}{2 \pi} \int_{-\pi}^{\pi} \frac{e^{i t}+z}{e^{i t}-z} \log \left|D\left(e^{i t}\right)\right| d t,
\end{aligned}
$$

where $\log \left|D\left(e^{i t}\right)\right| \in L^{1}(-\pi, \pi)$.

(ii) The measure $\sigma$ satisfies

$$
\operatorname{supp} \sigma \subset\left\{t \in[-\pi, \pi]: D\left(e^{i t}\right)=0\right\}
$$

\section{Remarks}

1. For the canonical factorisation of analytic functions see, for example, [23].

2. Note that for $D_{i n}(z)$ defined by $D_{i n}(z)=B(z) e^{-K_{\sigma}(z)}$, we have $\left|D_{\text {in }}(z)\right| \leqslant 1$, since $d \sigma \geqslant 0$ and $\operatorname{Re} \frac{e^{i t}+z}{e^{i t}-z} \geqslant 0$ for all $(t, z) \in \mathbb{T} \times \mathbb{D}$.

3. The closure of the set $\left\{z_{j}\right\} \cup \operatorname{supp} \sigma$ is called the spectrum of the inner function $D_{\text {in }}$.

4. $D_{B}=\frac{D}{B}$ has no zeros in the disk $\mathbb{D}$ and satisfies

$$
\log D_{B}(z)=\frac{1}{2 \pi} \int_{-\pi}^{\pi} \frac{e^{i t}+z}{e^{i t}-z} d \mu(t),
$$

where the measure $\mu$ equals

$$
d \mu(t)=\log \left|D\left(e^{i t}\right)\right| d t-d \sigma(t)
$$

Theorem 2.4 (Trace formulae) Let $V$ satisfy the condition (1.1). Then

$$
\begin{array}{r}
\frac{\sigma(\mathbb{T})}{2 \pi}-B_{0}=\frac{1}{2 \pi} \int_{-\pi}^{\pi} \log \left|D\left(e^{i t}\right)\right| d t \geqslant 0, \\
-d_{n}+B_{n}=\frac{1}{\pi} \int_{\mathbb{T}} e^{-i n t} d \mu(t), \quad n=1,2, \ldots,
\end{array}
$$

where $B_{0}=\log B(0)=\log \left(\prod_{j=1}^{N}\left|z_{j}\right|\right)<0$ and $B_{n}$ and $d_{n}$ are given by (2.11) and (2.5) respectively. In particular, 


$$
\begin{gathered}
\sum_{j=1}^{N}\left(\frac{1}{z_{j}}-\bar{z}_{j}\right)=\frac{2}{d} \operatorname{Tr} V+\frac{1}{\pi} \int_{\mathbb{T}} e^{-i t} d \mu(t), \\
\sum_{j=1}^{N}\left(\frac{1}{z_{j}^{2}}-\bar{z}_{j}^{2}\right)=\frac{4}{d^{2}} \operatorname{Tr} V^{2}+\frac{1}{\pi} \int_{\mathbb{T}} e^{-i 2 t} d \mu(t),
\end{gathered}
$$

and

$$
\begin{aligned}
\sum_{j=1}^{N} \operatorname{Im} \lambda_{j} & =\operatorname{Tr} \operatorname{Im} V-\frac{d}{2 \pi} \int_{\mathbb{T}} \sin t d \mu(t), \\
\sum_{j=1}^{N} \operatorname{Re} \sqrt{\lambda_{j}^{2}-d^{2}} & =\operatorname{Tr} \operatorname{Re} V+\frac{d}{2 \pi} \int_{\mathbb{T}} \cos t d \mu(t) .
\end{aligned}
$$

Theorem 2.5 Let $V$ satisfy the condition (1.1). Then we have the following estimates:

$$
\sum\left(1-\left|z_{j}\right|\right) \leqslant-B_{0} \leqslant C(d)\|V\|_{2 / 3}-\frac{\sigma(\mathbb{T})}{2 \pi} .
$$

If $\operatorname{Im} V \geqslant 0$, then

$$
\sum_{j=1}^{N} \operatorname{Im} \lambda_{j} \leqslant \operatorname{Tr} \operatorname{Im} V+C(d)\|V\|_{2 / 3}
$$

and if $V \geqslant 0$, then

$$
\sum_{j=1}^{N} \sqrt{\lambda_{j}^{2}-d^{2}} \leqslant \operatorname{Tr} V+C(d)\|V\|_{2 / 3}
$$

Remark Note that some of the results stated in Theorems 2.4 and 2.5 are new even for real-valued potentials, see Sect. 5 .

\section{Determinants}

\subsection{Properties of the Laplacian}

One may diagonalize the discrete Laplacian, using the (unitary) Fourier transform $\Phi: \ell^{2}\left(\mathbb{Z}^{d}\right) \rightarrow L^{2}\left(\mathbb{T}^{d}\right)$, where $\mathbb{T}=\mathbb{R} /(2 \pi \mathbb{Z})$. It is defined by

$$
(\Phi f)(k)=\widehat{f}(k)=\frac{1}{(2 \pi)^{\frac{d}{2}}} \sum_{n \in \mathbb{Z}^{d}} f(n) e^{i(n, k)}, \quad \text { where } \quad k=\left(k_{j}\right)_{j=1}^{d} \in \mathbb{T}^{d}
$$


In this "momentum representation" we have

$$
\Phi H \Phi^{*}=\widehat{\Delta}+\widehat{V}
$$

where the Laplacian is transformed into the multiplication operator

$$
(\widehat{\Delta} \widehat{f})(k)=h(k) \widehat{f}(k), \quad h(k)=\sum_{1}^{d} \cos k_{j}, \quad k \in \mathbb{T}^{d},
$$

and

$$
(\widehat{V} \widehat{f})(k)=\frac{1}{(2 \pi)^{\frac{d}{2}}} \int_{\mathbb{S}^{d}} \widehat{V}\left(k-k^{\prime}\right) \widehat{f}\left(k^{\prime}\right) d k^{\prime}
$$

Here

$$
\widehat{V}(k)=\frac{1}{(2 \pi)^{\frac{d}{2}}} \sum_{n \in \mathbb{Z}^{d}} V(n) e^{i(n, k)}, \quad V(n)=\frac{1}{(2 \pi)^{\frac{d}{2}}} \int_{\mathbb{T}^{d}} \widehat{V}(k) e^{-i(n, k)} d k .
$$

\subsection{Trace class operators}

Here for the sake of completeness we give some standard facts from Operator Theory in Hibert Spaces.

- Let $A, B$ be operators in Hilbert space $\mathcal{H}$ and let $A, B \in \mathcal{B}$ and $A B, B A \in \mathcal{B}_{1}$. Then

$$
\begin{aligned}
& \operatorname{Tr} A B=\operatorname{Tr} B A, \\
& \operatorname{det}(I+A B)=\operatorname{det}(I+B A) \text {. } \\
& |\operatorname{det}(I+A)| \leqslant e^{\|A\|_{\mathcal{B}_{1}}} \text {. } \\
& |\operatorname{det}(I+A)-\operatorname{det}(I+B)| \leqslant\|A-B\|_{\mathcal{B}_{1}} e^{1+\|A\|_{\mathcal{B}_{1}}+\|B\|_{\mathcal{B}_{1}}} \text {. }
\end{aligned}
$$

Moreover, $I+A$ is invertible if and only if $\operatorname{det}(I+A) \neq 0$.

- Suppose for a domain $\mathscr{D} \subset \mathbb{C}, \Omega(\cdot)-I: \mathscr{D} \rightarrow \mathcal{B}_{1}$ is analytic and invertible operator-valued function for any $z \in \mathscr{D}$. Then for $F(z)=\operatorname{det} \Omega(z)$ we have

$$
F^{\prime}(z)=F(z) \operatorname{Tr}\left(\Omega(z)^{-1} \Omega^{\prime}(z)\right)
$$

- For $K \in \mathcal{B}_{1}$ and $z \in \mathbb{C}$, the following identity holds true:

$$
\operatorname{det}(I-z K)=\exp \left(-\int_{0}^{z} \operatorname{Tr}\left(K(1-s K)^{-1}\right) d s\right)
$$

(see e.g. [19], p.167, or [32], p.331). 


\subsection{Fredholm determinant}

We recall here results from [21] about the asymptotics of the determinant $\mathcal{D}(\lambda)=$ $\operatorname{det}\left(I+V R_{0}(\lambda)\right)$ as $|\lambda| \rightarrow \infty$.

Lemma 3.1 Let $V \in \ell^{1}\left(\mathbb{Z}^{d}\right)$. Then the determinant $\mathcal{D}(\lambda)=\operatorname{det}\left(I+V R_{0}(\lambda)\right)$ is analytic in $\Lambda=\mathbb{C} \backslash[-d, d]$ and satisfies

$$
\mathcal{D}(\lambda)=1+O(1 / \lambda) \text { as }|\lambda| \rightarrow \infty
$$

uniformly in $\arg \lambda \in[0,2 \pi]$, and

$$
\begin{aligned}
& \log \mathcal{D}(\lambda)=-\sum_{n=1}^{\infty} \frac{(-1)^{n}}{n} \operatorname{Tr}\left(V R_{0}(\lambda)\right)^{n}, \\
& \log \mathcal{D}(\lambda)=-\sum_{n \geq 1} \frac{d_{n}}{n \lambda^{n}}, \quad d_{n}=\operatorname{Tr}\left(H^{n}-H_{0}^{n}\right),
\end{aligned}
$$

where the right-hand side is absolutely convergent for $|\lambda|>r_{1}$, with $r_{1}>0$ being a sufficiently large constant. In particular,

$$
\begin{aligned}
& d_{1}=\operatorname{Tr} V, \\
& d_{2}=\operatorname{Tr} V^{2} \\
& d_{3}=\operatorname{Tr}\left(V^{3}+6 d \tau^{2} V\right), \\
& d_{4}=\operatorname{Tr}\left(V^{4}+8 d \tau^{2} V^{2}+2 \tau^{2}\left(V_{\Delta}\right) V\right), \ldots,
\end{aligned}
$$

where $V_{\Delta}=\sum_{i=1}^{d}\left(S_{j} V S_{j}^{*}+S_{j}^{*} V S_{j}\right)$ and $\left(S_{j} f\right)(n)=f\left(n+e_{j}\right)$ and $\tau=\frac{1}{2}$.

Recall the conformal mapping $\lambda(\cdot): \mathbb{D} \rightarrow \Lambda$ given by $\lambda(z)=\frac{d}{2}\left(z+\frac{1}{z}\right),|z|<$ 1 , and note that $|\lambda| \rightarrow \infty$ iff $z \rightarrow 0$. We consider the operator-valued function $Y(\lambda(z)), \lambda \in \Lambda$, defined by

$$
Y(\lambda(z))=V_{2} X(\lambda(z)), \quad X(\lambda(z))=\left|V_{1}\right|^{1 / 2} R_{0}(\lambda(z))\left|V_{1}\right|^{1 / 2} V_{1}\left|V_{1}\right|^{-1},
$$

where $V_{1}$ and $V_{2}$ are defined in (1.2).

Theorem 3.2 Let $V$ satisfy the condition (1.1). Then the operator-valued function $Y(\lambda(z)): \mathbb{D} \rightarrow \mathcal{B}_{1}$ is analytic in the unit disc $\mathbb{D}$, Höder up to the boundary and satisfies

$$
\|Y(\lambda(z))\|_{\mathcal{B}_{1}} \leqslant C(d)\|V\|_{2 / 3}, \quad \forall z \in \mathbb{D} .
$$

Moreover, the function $D(z), z \in \mathbb{D}$ belongs to $\mathscr{H}_{\infty}$, Höder up to the boundary and

$$
\|D(\cdot)\|_{\mathscr{H}_{\infty}} \leqslant e^{C(d)\|V\|_{2 / 3}} .
$$


Proof The operator $V_{2}$ belongs to $\mathcal{B}_{2}$ and due to Theorem 7.3 (see "Appendix 2") the operator-valued function $X(\cdot): \Lambda \rightarrow \mathcal{B}_{2}$ is analytic in $\Lambda$, Höder up to the boundary and satisfies the inequality

$$
\|X(\lambda)\|_{\mathcal{B}_{2}} \leqslant C(d)\left\|V_{1}\right\|_{2}, \quad \forall \lambda \in \Lambda=\mathbb{C} \backslash[-d, d]
$$

Thus the operator-valued function $Y(\lambda(z))=V_{2} X(\lambda(z)): \mathbb{D} \rightarrow \mathcal{B}_{1}$ is of trace class. Moreover, the function $D(z), z \in \mathbb{D}$, is analytic in the unit disc $\mathbb{D}$, Höder up to the boundary and due to (3.2) it satisfies (3.3).

The function $D(z)=\mathcal{D}(\lambda(z))$ is analytic in $\mathbb{D}$ whose zeros are given by

$$
z_{j}=z\left(\lambda_{j}\right), \quad j=1,2, \ldots, N
$$

where $\lambda_{j}$ are zeros (counting with multiplicity) of $\mathcal{D}(\lambda)$ in $\Lambda=\mathbb{C} \backslash[-d, d]$, i.e., eigenvalues of $H$ (counted with algebraic multiplicity).

Lemma 3.3 Let a potential $V \in \ell^{1}\left(\mathbb{Z}^{d}\right)$ and let the branch of $\log D(z)$ be defined by the condition $\log D(0)=0$. Then $\log D(z)$ is analytic in $\mathbb{D}_{r_{0}}$, where $r_{0}$ is given by (2.2), and has the following Taylor expansion

$$
\log D(z)=-c_{1} z-c_{2} z^{2}-c_{3} z^{3}-c_{4} z^{4}-\ldots, \text { as }|z|<r_{0},
$$

and

$$
c_{1}=d_{1} a, \quad c_{2}=d_{2} a^{2}, \quad c_{3}=d_{3} a^{3}-c_{1}, \quad c_{4}=d_{4} a^{4}-c_{2}, \ldots
$$

where $a=\frac{2}{d}$ and the coefficients $d_{j}$ are given by (3.2).

Proof We have

$$
\begin{aligned}
\frac{1}{\lambda} & =\frac{a z}{1+z^{2}}=a\left(z-z^{3}+O\left(z^{5}\right)\right), \quad \frac{1}{\lambda^{2}}=a^{2}\left(z^{2}-z^{4}+O\left(z^{6}\right)\right), \\
\frac{1}{\lambda^{3}} & =a^{3} z^{3}+O\left(z^{5}\right),
\end{aligned}
$$

as $z \rightarrow 0$. Substituting these asymptotics into (3.1) we obtain (3.4) and (3.5).

\section{Proof of the main results}

We are ready to prove main results.

Proof of Theorem 2.1 By Theorem 3.2 the determinant $D(z), z \in \mathbb{D}$, is analytic and Höder up to the boundary. In particular, $D \in \mathscr{H}_{\infty}$. Moreover, Lemma 3.1 gives (2.3) and Lemma 3.3 implies (2.4)-(2.5). 
Proof of Theorem 2.2 Due to Theorem 2.1 the determinant $D(z)$ is analytic in $\mathbb{D}$ and Höder up to the boundary. Then Theorem 6.2 (see "Appendix 1") yields

$$
\mathcal{Z}_{D}:=\sum_{j=1}^{\infty}\left(1-\left|z_{j}\right|\right)<\infty
$$

and the Blaschke product $B(z)$ given by

$$
B(z)=\prod_{j=1}^{N} \frac{\left|z_{j}\right|}{z_{j}} \frac{z_{j}-z}{1-\bar{z}_{j} z}, \quad z \in \mathbb{D}
$$

converges absolutely for $|z|<1$. We have $D(z)=B(z) D_{B}(z)$, where $D_{B}$ is analytic in the unit disc $\mathbb{D}$ and has no zeros in $\mathbb{D}$. Thus we have proved (ii). (i) Equalities (2.9) and (2.10) follow from 6.1. (iii) For small a sufficiently small $z$ and for $t=z_{j} \in \mathbb{D}$ for some $j$, we have the following identity:

$$
\log \frac{|t|}{t} \frac{t-z}{1-\bar{t} z}=\log |t|+\log \left(1-\frac{z}{t}\right)-\log (1-\bar{t} z)=\log |t|-\sum_{n \geqslant 1}\left(\frac{1}{t^{n}}-\bar{t}^{n}\right) \frac{z^{n}}{n} .
$$

Besides,

$$
\begin{aligned}
\left.|1-| t\right|^{n} \mid & \leqslant n|1-| t||, \quad\left|t^{-n}-\bar{t}^{n}\right| \leqslant\left|1-t^{n}\right|+\left|1-t^{-n}\right| \\
& \leqslant\left|1-t^{n}\right|\left(1+\frac{1}{|t|^{n}}\right) \leqslant\left.|1-| t\right|^{n}\left|\frac{2}{r_{0}^{n}} \leqslant\right| 1-|t| \mid \frac{2 n}{r_{0}^{n}},
\end{aligned}
$$

where $r_{0}=\inf \left|z_{j}\right|>0$. This yields

$$
\begin{aligned}
\log B(z) & =\sum_{j=1}^{N} \log \left(\frac{\left|z_{j}\right|}{z_{j}} \frac{z_{j}-z}{1-\bar{z}_{j} z}\right)=\sum_{j=1}^{N}\left(\log \left|z_{j}\right|+\log \left(1-\left(z / z_{j}\right)\right)-\log \left(1-\bar{z}_{j} z\right)\right) \\
& =\sum_{j=1}^{N} \log \left|z_{j}\right|-\sum_{n=1}^{\infty} \sum_{j=1}^{N}\left(\frac{1}{z_{j}^{n}}-\bar{z}_{j}^{n}\right) \frac{z^{n}}{n}=\log B(0)-b(z) .
\end{aligned}
$$

Here

$$
b(z)=\sum_{n=1}^{N} \sum_{j=1}^{\infty}\left(\frac{1}{z_{j}^{n}}-\bar{z}_{j}^{n}\right) \frac{z^{n}}{n}=\sum_{n=1}^{N} z^{n} B_{n}, \quad B_{n}=\frac{1}{n} \sum_{j=1}^{N}\left(\frac{1}{z_{j}^{n}}-\bar{z}_{j}^{n}\right),
$$

where the function $b$ is analytic in the disk $\left\{|z|<\frac{r_{0}}{2}\right\}$ and $B_{n}$ satisfy

$$
\left|B_{n}\right| \leqslant \frac{1}{n} \sum_{j=1}^{N}\left|\frac{1}{z_{j}^{n}}-\bar{z}_{j}^{n}\right| \leqslant \frac{2}{r_{0}^{n}} \sum_{j=1}^{N}|1-| z_{j}||=\frac{2}{r_{0}^{n}} \mathcal{Z}_{D},
$$


where $\mathcal{Z}_{D}:=\sum_{j=1}^{\infty}\left(1-\left|z_{j}\right|\right)$. Thus

$$
|b(z)| \leqslant \sum_{n=1}^{\infty}\left|B_{n}\right||z|^{n} \leqslant 2 \mathcal{Z}_{D} \sum_{n=1}^{\infty} \frac{|z|^{n}}{r_{0}^{n}}=\frac{2 \mathcal{Z}_{D}}{1-\frac{|z|}{r_{0}}} .
$$

Proof of Theorem 2.3. (i) Theorem 2.1 implies $D \in \mathscr{H}_{\infty}$. Therefore the canonical representation (2.12) follows from Lemma 6.3. (ii) The relation (6.3) gives the proof of (ii), since due to Theorem 2.1 D is analytic and Höder up to the boundary.

Proof of Theorem 2.4. By using Lemma 6.4, (2.3)-(2.5) and Proposition 2.2 we obtain identities (2.14)-(2.16).

We have the following identities for $z \in \mathbb{D}$ and $\zeta=\frac{\lambda}{d} \in \Lambda_{1}$ :

$$
2 \zeta=z+\frac{1}{z}, \quad z=\zeta-\sqrt{\zeta^{2}-1}, \quad z-\frac{1}{z}=-2 \sqrt{\zeta^{2}-1} .
$$

These identities yield

$$
\begin{aligned}
& \bar{z}-\frac{1}{z}=z+\bar{z}-2 \zeta=2 \operatorname{Re} z-2 \zeta \\
& \bar{z}-\frac{1}{z}=\bar{z}-z-2 \sqrt{\zeta^{2}-1}=-2 i \operatorname{Im} z-2 \sqrt{\zeta^{2}-1}
\end{aligned}
$$

Then we obtain

$$
\begin{aligned}
& \frac{2}{d} \operatorname{Tr} \operatorname{Im} V+\operatorname{Im} \sum_{j=1}^{N}\left(\bar{z}_{j}-\frac{1}{z_{j}}\right)=\frac{1}{\pi} \int_{\mathbb{T}} \sin t d \mu(t), \\
& \operatorname{Im}\left(\bar{z}_{j}-\frac{1}{z_{j}}\right)=-2 \operatorname{Im} \zeta_{j}=-\frac{2}{d} \operatorname{Im} \lambda_{j}, \\
& \operatorname{Re}\left(\bar{z}_{j}-\frac{1}{z_{j}}\right)=2 \operatorname{Re}\left(z_{j}-\zeta_{j}\right)=-2 \operatorname{Re} \sqrt{\zeta_{j}^{2}-1}
\end{aligned}
$$

and thus

$$
\begin{aligned}
& \sum_{j=1}^{N} \operatorname{Im} \lambda_{j}=\operatorname{Tr} \operatorname{Im} V-\frac{d}{2 \pi} \int_{\mathbb{T}} \sin t d \mu(t), \\
& \sum_{j=1}^{N} \operatorname{Re} \sqrt{\lambda_{j}^{2}-d^{2}}=\operatorname{Tr} \operatorname{Re} V+\frac{d}{2 \pi} \int_{\mathbb{T}} \cos t d \mu(t) .
\end{aligned}
$$

Proof of Theorem 2.5. The simple inequality $1-x \leqslant-\log x$ for $\forall x \in(0,1]$, implies $-B_{0}=-B(0)=-\sum \log \left|z_{j}\right| \geqslant \sum\left(1-\left|z_{j}\right|\right)$. Then substituting the last estimate and the estimate (2.3) into the first trace formula (2.14) we obtain (2.19). 
In order to determine the next two estimates we use the trace formula (2.14). Let $\operatorname{Im} V \geqslant 0$. Then $\operatorname{Im} \lambda_{j} \geqslant 0$ and the estimates (2.3) and (2.19) and the second trace formula (2.18) imply

$$
\begin{aligned}
\sum_{j=1}^{N} \operatorname{Im} \lambda_{j}-\operatorname{Tr} \operatorname{Im} V & =-\frac{d}{2 \pi} \int_{\mathbb{T}} \sin t d \mu(t) \\
& \leqslant \frac{d}{2 \pi} \int_{\mathbb{T}}\left(C\|V\|_{\frac{2}{3}} d t+d \sigma(t)\right) \leqslant C(d)\|V\|_{\frac{2}{3}},
\end{aligned}
$$

which yields (2.20). Similar arguments give (2.21).

\section{Schrödinger operators with real potentials}

Consider Schrödinger operators $H=-\Delta+V$, where the potential $V$ is real and satisfies the condition (1.1). The spectrum $\sigma(H)$ of $H$ equals

$$
\sigma(H)=\sigma_{a c}(H) \cup \sigma_{s c}(H) \cup \sigma_{p}(H) \cup \sigma_{d i s}(H), \quad \sigma_{a c}(H)=[-d, d],
$$

where

$$
\sigma_{p}(H) \subset[-d, d], \quad \sigma_{d i s}(H) \subset \mathbb{R} \backslash[-d, d] .
$$

Note that each eigenvalues of $H$ has a finite multiplicity.

\subsection{Discrete spectrum}

Let us label the discrete eigenvalues

$$
\cdots \leqslant \lambda_{-2} \leqslant \lambda_{-1}<-d<d<\lambda_{1} \leqslant \lambda_{2} \leqslant \cdots
$$

The corresponding points $z_{j} \in \mathbb{D}$ are real and satisfy

$$
\lambda_{j}=\frac{d}{2}\left(z_{j}+\frac{1}{z_{j}}\right), \quad j \in \mathbb{Z} \backslash\{0\} .
$$

Moreover, we have the identity

$$
\sqrt{\lambda^{2}-d^{2}}=\frac{d}{2}\left(z-\frac{1}{z}\right)
$$

for all $\lambda \in \Lambda$ and $z \in \mathbb{D}$. If $\lambda$ is the eigenvalue of $H$, then we have

$$
\begin{gathered}
\frac{d}{2}\left(\frac{1}{z}-z\right)=-\left|\lambda^{2}-d^{2}\right|^{\frac{1}{2}} \quad \text { if } \quad \lambda<-d, \\
\frac{d}{2}\left(\frac{1}{z}-z\right)=\left|\lambda^{2}-d^{2}\right|^{\frac{1}{2}} \quad \text { if } \quad \lambda>d .
\end{gathered}
$$


The next result follows immediately from Theorem 2.4.

Theorem 5.1 (The trace formulas) Let a real potential $V$ satisfy the condition (1.1). Then the spectral data of the operator $H$ and the potential $V$ satisfy the infinite number of trace formulae

$$
\begin{aligned}
0 \leqslant & \frac{\sigma(\mathbb{T})}{2 \pi}-B_{0}=\frac{1}{2 \pi} \int_{\mathbb{T}} \log \left|D\left(e^{i t}\right)\right| d t \leqslant C(d, p)\|V\|_{2 / 3}, \\
& -\operatorname{Tr} V+\sum_{j=1}^{N}\left|\lambda_{j}^{2}-d^{2}\right|^{\frac{1}{2}} \operatorname{sign} \lambda_{j}=\frac{d}{2 \pi} \int_{\mathbb{T}} e^{-i t} d \mu(t), \\
& -\operatorname{Tr} V^{2}+\sum_{j=1}^{N}\left|\lambda_{j}\right|\left|\lambda_{j}^{2}-d^{2}\right|^{\frac{1}{2}}=\frac{d^{2}}{4 \pi} \int_{\mathbb{T}} e^{-i 2 t} d \mu(t), \ldots
\end{aligned}
$$

Proof We have the following simple formula of the zeros $z_{j}$ of the determinant $D$ in terms of the eigenvalue $\lambda_{j}$ of $H$ :

$$
z=\frac{1}{d}\left(\lambda \pm \sqrt{\lambda^{2}-d^{2}}\right)
$$

Using this identity and trace formulae from Theorem 2.4 we obtain (5.2) and (5.2).

Corollary 5.2 Let a potential $V$ be real and satisfy the condition (1.1). Then the following estimates hold:

$$
\begin{gathered}
\sum_{\lambda_{j}} \frac{\sqrt{\left|\lambda_{j}\right|-d}}{\sqrt{\left|\lambda_{j}\right|+d}} \leqslant C_{d}\|V\|_{2 / 3}, \\
\sum_{j=1}^{N}\left|\lambda_{j}\right|\left|\lambda_{j}^{2}-d^{2}\right|^{\frac{1}{2}} \leqslant \operatorname{Tr} V^{2}+\frac{d^{2}}{4 \pi} C(d)\|V\|_{2 / 3} .
\end{gathered}
$$

Proof We consider the case (5.2). If $\lambda>d$, then we have $z \in(0,1)$ and then

$$
\begin{aligned}
-\log z & \geqslant 1-z=1-\frac{1}{d}\left(\lambda-\sqrt{\lambda^{2}-d^{2}}\right)=\frac{1}{d}\left(d-\lambda+\sqrt{\lambda^{2}-d^{2}}\right) \\
& =\frac{\sqrt{\lambda-d}}{d}(\sqrt{\lambda+d}-\sqrt{\lambda-d})=\frac{2 \sqrt{\lambda-d}}{(\sqrt{\lambda+d}+\sqrt{\lambda-d})} \geqslant \frac{\sqrt{\lambda-d}}{\sqrt{\lambda+d}} .
\end{aligned}
$$

We have the same estimates for the case $\lambda<-d$ and $z \in(-1,0)$. This yields

$$
\sum_{\lambda_{j}>d} \frac{\sqrt{\lambda_{j}-d}}{\sqrt{\lambda_{j}+d}}+\sum_{\lambda_{j}<-d} \frac{\sqrt{-\lambda_{j}-d}}{\sqrt{-\lambda_{j}+d}}=\sum_{\lambda_{j}} \frac{\sqrt{\left|\lambda_{j}\right|-d}}{\sqrt{\left|\lambda_{j}\right|+d}} \leqslant C_{d}\|V\|_{\frac{2}{3}},
$$

that gives (5.4). The proof of (5.5) is similar. 


\section{Appendix 1: Hardy spaces}

\subsection{Analytic functions}

In order to obtain trace formulae stated in Theorem 2.4 we need to recall the basic facts from Theory of Analytic Functions in the unit disc.

The following fact can be found for example in [18], Sect. 2.

Lemma 6.1 Let $\left\{z_{j}\right\}$ be a sequence of points in $\mathbb{D} \backslash\{0\}$ such that $\sum\left(1-\left|z_{j}\right|\right)<\infty$ and let $m \geqslant 0$ be an integer. Then the Blaschke product

$$
B(z)=z^{m} \prod_{z_{j} \neq 0} \frac{\left|z_{j}\right|}{z_{j}}\left(\frac{z_{j}-z}{1-\bar{z}_{j} z}\right),
$$

converges in $\mathbb{D}$. Moreover, the function $B$ is in $\mathscr{H}_{\infty}$ and zeros of $B$ are precisely the points $z_{j}$, according to the multiplicity. Moreover,

$$
|B(z)| \leqslant 1 \quad \forall z \in \mathbb{D},
$$

$$
\begin{gathered}
\lim _{r \rightarrow 1}\left|B\left(r e^{i \vartheta}\right)\right|=\left|B\left(e^{i \vartheta}\right)\right|=1 \quad \text { almost everywhere, } \vartheta \in \mathbb{T}, \\
\lim _{r \rightarrow 1} \int_{0}^{2 \pi} \log \left|B\left(r e^{i \vartheta}\right)\right| d \vartheta=0 .
\end{gathered}
$$

For the next statement see for example in Koosis [23], page 67.

Theorem 6.2 Let $f$ be analytic in the unit disc $\mathbb{D}$ and let $z_{j} \neq 0, j=1,2, \ldots, N \leqslant$ $\infty$ be its zeros labeled by

$$
0<\left|z_{1}\right| \leqslant \cdots \leqslant\left|z_{j}\right| \leqslant\left|z_{j+1} \leqslant\right| z_{j+2} \mid \leqslant \cdots
$$

Suppose that $f$ satisfies the condition

$$
\sup _{r \in(0,1)} \int_{0}^{2 \pi} \log \left|f\left(r e^{i \vartheta}\right)\right| d \vartheta<\infty .
$$

Then

$$
\sum_{j=1}^{N}\left(1-\left|z_{j}\right|\right)<\infty
$$

The Blaschke product $B(z)$ given by

$$
B(z)=z^{m} \prod_{j=1}^{N} \frac{\left|z_{j}\right|}{z_{j}} \frac{\left(z_{j}-z\right)}{\left(1-\bar{z}_{j} z\right)},
$$


where $m$ is the multiplicity of $B$ at zero, converges absolutely for $\{|z|<1\}$. Besides, $f_{B}(z)=f(z) / B(z)$ is analytic in the unit disc $\mathbb{D}$ and has no zeros in $\mathbb{D}$.

Moreover, if $f(0) \neq 0$ and if $u(z)$ is the least harmonic majorant of $\log |f(z)|$, then

$$
\sum\left(1-\left|z_{j}\right|\right)<u(0)-\log |f(0)|
$$

We now remind the facts regarding the canonical representation (6.2) for a function $f \in \mathscr{H}_{\infty}$ (see, [23], p. 76).

Lemma 6.3 Let a function $f \in \mathscr{H}_{\infty}$. Let $B$ be its Blaschke product. Then there exists a singular measure $\sigma=\sigma_{f} \geqslant 0$ on $[-\pi, \pi]$ with

$$
\begin{aligned}
f(z) & =B(z) e^{i c-K_{\sigma}(z)} e^{K_{f}(z)}, \\
K_{\sigma}(z) & =\frac{1}{2 \pi} \int_{-\pi}^{\pi} \frac{e^{i t}+z}{e^{i t}-z} d \sigma(t), \\
K_{f}(z) & =\frac{1}{2 \pi} \int_{-\pi}^{\pi} \frac{e^{i t}+z}{e^{i t}-z} \log \left|f\left(e^{i t}\right)\right| d t,
\end{aligned}
$$

for all $|z|<1$, where $c$ is real constant and $\log \left|f\left(e^{i t}\right)\right| \in L^{1}(-\pi, \pi)$.

We define the functions (after Beurling) in the disc by

$$
\begin{aligned}
& f_{\text {in }}(z)=B(z) e^{i c-K_{\sigma}(z)} \text { the inner factor of } f \\
& f_{\text {out }}(z)=e^{K_{f}(z)} \text { the outer factor of } f
\end{aligned}
$$

for $|z|<1$. Note that we have $\left|f_{\text {in }}(z)\right| \leqslant 1$, since $d \sigma \geqslant 0$.

Thus $f_{B}(z)=\frac{f(z)}{B(z)}$ has no zeros in the disc $\mathbb{D}$ and satisfies

$$
\log f_{B}(z)=i c+\frac{1}{2 \pi} \int_{-\pi}^{\pi} \frac{e^{i t}+z}{e^{i t}-z} d \mu(t),
$$

where the measure $\mu$ equals

$$
d \mu(t)=\log \left|f\left(e^{i t}\right)\right| d t-d \sigma(t) .
$$

For a function $f$ continuous on the disc $\overline{\mathbb{D}}$ we define the set of zeros of $f$ lying on the boundary $\partial \mathbb{D}$ by

$$
\mathfrak{S}_{0}(f)=\{z \in \mathbb{T}: f(z)=0\} .
$$

It is well known that the support of the singular measure $\sigma=\sigma_{f}$ satisfies

$$
\operatorname{supp} \sigma_{f} \subset \mathfrak{S}_{0}(f)=\{z \in \mathbb{T}: f(z)=0\},
$$


see for example, Hoffman [20], p. 70.

In the next statement we consider trace formulae for a function $f \in \mathscr{H}_{\infty}$.

Lemma 6.4 Let $f \in \mathscr{H}_{\infty}$ and $f(0)=1$ and let $B$ be its Blaschke product. Assume that the functions $\log f$ and $F=\log f_{B}$ have the Taylor series in the disc $\mathbb{D}_{r} \subset \mathbb{D}$, $r>0$, given by

$$
\begin{aligned}
\log f(z) & =-f_{1} z-f_{2} z^{2}-f_{3} z^{3}-\cdots, \\
F & =\log f_{B}(z)=F_{0}+F_{1} z+F_{2} z^{2}+F_{3} z^{3}+\cdots, \\
\log B(z) & =B_{0}-B_{1} z-B_{2} z^{2}-\ldots, \quad \text { as } z \rightarrow 0, \\
F_{0} & =-\log B(0)>0, \quad F_{n}=B_{n}-f_{n}, \quad n \geqslant 1 .
\end{aligned}
$$

Then the factorization (6.2) holds true and we have

$$
\begin{aligned}
& c=0, \quad F_{0}=-\log B(0)=\frac{\mu(\mathbb{T})}{2 \pi} \geqslant 0, \\
& F_{n}=\frac{1}{\pi} \int_{-\pi}^{\pi} e^{-i n t} d \mu(t), \quad n=1,2, \ldots,
\end{aligned}
$$

where the measure $d \mu(t)=\log \left|f\left(e^{i t}\right)\right| d t-d \sigma(t)$.

Proof By using (6.2) we find

$$
1=f(0)=B(0) e^{i c-K_{\sigma}(0)} e^{K_{f}(0)} .
$$

Since $B(0), K_{\sigma}(0), K_{f}(0)$ and $c$ are real we obtain $c=0$. Moreover, the inequality (6.1) implies $F_{0} \geqslant 0$. In order to show (6.6) we consider the asymptotic behaviour of the Schwatz integral

$$
f(z)=B(z) f_{B}(z), \quad F(z)=\log f_{B}(z)=\frac{1}{2 \pi} \int_{-\pi}^{\pi} \frac{e^{i t}+z}{e^{i t}-z} d \mu(t),
$$

as $z \rightarrow 0$. Indeed,

$$
\frac{e^{i t}+z}{e^{i t}-z}=1+\frac{2 z e^{-i t}}{1-z e^{-i t}}=1+2 \sum_{n \geqslant 1}\left(z e^{-i t}\right)^{n}=1+2\left(z e^{-i t}\right)+2\left(z e^{-i t}\right)^{2}+\cdots
$$

Thus (6.7), (6.8) yield the Taylor series at $z=0$ :

$$
\frac{1}{2 \pi} \int_{-\pi}^{\pi} \frac{e^{i t}+z}{e^{i t}-z} d \mu(t)=\frac{\mu(\mathbb{T})}{2 \pi}+\mu_{1} z+\mu_{2} z^{2}+\mu_{3} z^{3}+\mu_{4} z^{4}+\cdots \quad \text { as } \quad z \rightarrow 0,
$$

where

$$
\mu(\mathbb{T})=\int_{0}^{2 \pi} d \mu(t), \quad \mu_{n}=\frac{1}{\pi} \int_{0}^{2 \pi} e^{-i n \vartheta} d \mu(t), \quad n \in \mathbb{Z}
$$


Thus comparing (6.4) and (6.9) we obtain

$$
-\log B(0)=\frac{\mu(\mathbb{T})}{2 \pi} \geqslant 0, F_{n}=\mu_{n} \quad \forall n \geqslant 1 .
$$

\section{Appendix 2: Estimates involving Bessel's functions}

In order to complete the proof of Theorem 3.2 we need some uniform estimates for the Bessel functions $J_{m}, m \in \mathbb{Z}$, with respect to $m$. We use the integral representation

$$
J_{m}(t)=\frac{1}{2 \pi} \int_{0}^{2 \pi} e^{-i m k-i t \sin k} d k=\frac{i^{m}}{2 \pi} \int_{0}^{2 \pi} e^{-i m k+i t \cos k} d k .
$$

Note that for all $(t, m) \in \mathbb{R} \times \mathbb{Z}$ :

$$
J_{-m}(t)=J_{m}(t) \text { and } J_{m}(-t)=(-1)^{m} J_{n}(t) .
$$

Our estimates are based on the following three asymptotic formulae, see [36], Ch IV, $\S 2$.

Let $\xi=\frac{m}{t}$. Then for a fixed $\varepsilon, 0<\varepsilon<1$ we have:

1. If $\xi>1+\varepsilon$ then

$$
J_{m}(t)=\frac{1}{2} \sqrt{\frac{2}{\pi t}} \frac{1}{\left(\xi^{2}-1\right)^{1 / 4}} e^{-t\left(\xi \ln \left(\xi+\sqrt{\xi^{2}-1}\right)-\sqrt{\xi^{2}-1}\right)}\left(1+O\left(\frac{1}{m}\right)\right) .
$$

Therefore if $\xi>1+\varepsilon$ then this formula implies the uniform with respect to $m$ exponential decay of the Bessel function in $t$.

2. If $\xi<1-\varepsilon$ then

$$
\begin{aligned}
J_{m}(t)= & \frac{1}{2} \sqrt{\frac{2}{\pi t}} \frac{1}{\left(\xi^{2}-1\right)^{1 / 4}}\left(e^{-i \pi / 4 i+i t\left(-\xi \arccos \xi+\sqrt{1-\xi^{2}}\right)}\right. \\
& \left.+e^{i \pi / 4 i+i t\left(\xi \arccos \xi-\sqrt{1-\xi^{2}}\right)}\right)\left(1+O\left(\frac{1}{t}\right)\right) .
\end{aligned}
$$

In this case the Bessel function oscillates as $t \rightarrow \infty$ and obviously the latter formula implies the uniform with respect to $m$ estimate

$$
\left|J_{m}(t)\right| \leqslant C t^{-1 / 2}, \quad C=C(\varepsilon) .
$$

3. We now consider the third case $1-\varepsilon \leqslant \xi \leqslant 1+\varepsilon$ that is more complicated.

Lemma 7.1 If $1-\varepsilon \leqslant \xi \leqslant 1+\varepsilon, \varepsilon>0$, then there is a constant $C=C(\varepsilon)$ such that

$$
J_{m}(t) \leqslant C t^{-\frac{1}{4}}\left(|t|^{\frac{1}{3}}+|m-t|\right)^{-\frac{1}{4}}, \quad \forall m, t, \quad|m-t|<\varepsilon t .
$$


Proof If $1-\varepsilon \leqslant \xi \leqslant 1+\varepsilon$, then (see [36], Ch IV, $\S 2$ )

$$
\begin{aligned}
J_{m}(t)= & \frac{v\left(t^{2 / 3} \tau(\xi)\right)}{t^{1 / 3}}\left(c_{0}(\xi)+O\left(\frac{1}{t}\right)\right) \\
& +\frac{v^{\prime}\left(t^{2 / 3} \tau(\xi)\right)}{t^{4 / 3}}\left(d_{0}(\xi)+O\left(\frac{1}{t}\right)\right),
\end{aligned}
$$

where $v$ is the Airy function and

$$
\tau^{3 / 2}(\xi)=\xi \ln \left(\xi+\sqrt{\xi^{2}-1}\right)-\sqrt{\xi^{2}-1}
$$

and therefore

$$
\tau(\xi)=2^{1 / 3}(\xi-1)+O\left((\xi-1)^{2}\right), \quad \text { as } \quad \xi \rightarrow 1 .
$$

Besides the functions $c_{0}(\xi)$ and $d_{0}(\xi)$ are bounded with respect to $\xi$ and, for example,

$$
c_{0}(\xi)=\sqrt{\frac{2}{\pi}}\left(\frac{\tau(\xi)}{\xi^{2}-1}\right)^{1 / 4},
$$

(see [29] formulae (10.06), (10.07))

In what follows all the constants depend on $\varepsilon, 0<\varepsilon<1$, but not on $m$ and $t$. Due (7.7) there are constants $c$ and $C$ such that

$$
c(\varepsilon)(1+|y|)^{\frac{1}{4}} \leqslant\left(t^{\frac{1}{3}}+|m-t|\right)^{\frac{1}{4}} t^{-\frac{1}{12}} \leqslant C(\varepsilon)(1+|y|)^{\frac{1}{4}},
$$

where $y=t^{\frac{2}{3}} \tau(\xi)$. Moreover, since $|\xi-1|=\left|\frac{m}{t}-1\right| \leqslant \varepsilon$ we also have

$$
\left(t^{\frac{1}{3}}+|m-t|\right)^{\frac{1}{4}} t^{-\frac{1}{12}} \leqslant C(\varepsilon) t^{\frac{1}{6}} .
$$

Applying estimates for the Airy functions in (7.6)

$$
|v(y)| \leqslant C(1+|y|)^{-1 / 4}, \quad\left|v^{\prime}(y)\right| \leqslant C(1+|y|)^{1 / 4}
$$

and using (7.8), (7.9) we find that if $|t| \geqslant 1$

$$
\begin{aligned}
\left|J_{m}(t)\right| & \leqslant C\left(\frac{1}{(1+|y|)^{\frac{1}{4}}|t|^{\frac{1}{3}}}+\frac{(1+|y|)^{\frac{1}{4}}}{|t|^{\frac{4}{3}}}\right) \\
& \leqslant C\left(\frac{t^{\frac{1}{12}}}{t^{\frac{1}{3}}\left(t^{\frac{1}{3}}+|m-t|\right)^{\frac{1}{4}}}+\frac{\left(t^{\frac{1}{3}}+|m-t|\right)^{\frac{1}{4}}}{t^{\frac{4}{3}} t^{\frac{1}{12}}}\right) \\
& \leqslant C t^{-\frac{1}{4}}\left(|t|^{\frac{1}{3}}+|m-t|\right)^{-\frac{1}{4}} .
\end{aligned}
$$


The proof is complete.

Let us now consider the operator $e^{i t \Delta}, t \in \mathbb{R}$. It is unitary on $L^{2}\left(\mathbb{S}^{d}\right)$ and its kernel is given by

$$
\left(e^{i t \Delta}\right)\left(n-n^{\prime}\right)=\frac{1}{(2 \pi)^{d}} \int_{\mathbb{S}^{d}} e^{-i\left(n-n^{\prime}, k\right)+i t h(k)} d k, \quad n, n^{\prime} \in \mathbb{Z}^{d},
$$

where $h(k)=\sum_{j=1}^{d} \cos k_{j}, k=\left(k_{j}\right)_{j=1}^{d} \in \mathbb{S}^{d}$.

Lemma 7.2 Let $n=\left(n_{j}\right)_{j=1}^{d} \in \mathbb{Z}^{d}, d \geqslant 1$. Then

$$
\left(e^{i t \Delta}\right)(n)=i^{-|n|} \prod_{j=1}^{d} J_{n_{j}}(t), \quad(n, t) \in \mathbb{Z}^{d} \times \mathbb{R}
$$

where $|n|=\left|n_{1}\right|+\cdots+\left|n_{d}\right|$. Moreover, the following estimates are satisfied:

$$
\begin{array}{r}
\left|\left(e^{i t \Delta}\right)(n)\right| \leqslant C_{1}|t|^{-\frac{d}{3}}, \quad t \geqslant 1, \\
\int_{1}^{\infty} t^{\gamma}\left|J_{m}(t)\right|^{d} d t<C_{2}, \quad \text { if } m \in \mathbb{Z}, d \geqslant 3,
\end{array}
$$

for all $(t, n) \in \mathbb{R} \times \mathbb{Z}^{d}$ and any $\gamma \in\left[0, \frac{1}{6}\right]$ and some constants $C_{1}=C_{1}(d)$ and $C_{2}=C_{2}(d)$.

Proof Let $d=1$ and $h(k)=\cos k, k \in \mathbb{T}$. Then using (7.10) and (7.1) we obtain

$$
\left(e^{i t \Delta}\right)(n)=\frac{1}{(2 \pi)} \int_{\mathbb{T}} e^{-i n k+i t \cos k} d k=i^{-n} J_{n}(t), \quad \forall(n, t) \in \mathbb{Z} \times \mathbb{R}
$$

which yields (7.11) for $d=1$. Due to the separation of variables we also obtain (7.11) for any $d \geqslant 1$.

In view of (7.2) it is enough to consider the case $n_{j} \geqslant 0$. In order to obtain (7.12) it is enough ti apply the inequalities (7.3), (7.4) and also (7.5) if in this inequality we ignore the term $|m-t|$.

If $d>3$, then (7.12) implies (7.12). Let now $d=3$. From (7.3)-(7.5) we obtain

$$
\begin{aligned}
& \int_{1}^{\infty}\left|J_{m}(t)\right|^{d} d t \leqslant C\left(\int_{1}^{\infty}|t|^{-3 / 2} d t+\int_{1}^{\infty}|t|^{-\frac{3}{4}}\left(|t|^{\frac{1}{3}}+|m-t|\right)^{-\frac{3}{4}} d t\right) \\
& \leqslant C / 2+C \int_{1}^{\infty}|t|^{-\frac{3}{4}}(1+|m-t|)^{-\frac{3}{4}} d t \\
& \leqslant C / 2+C\left(\int_{1}^{\infty}|t|^{-3 / 2} d t\right)^{1 / 2}\left(\int_{1}^{\infty}(1+|m-t|)^{-3 / 2} d t\right)^{1 / 2}<\infty .
\end{aligned}
$$

The proof for $\gamma \in\left[0, \frac{1}{6}\right]$ is similar. 
Theorem 7.3 (i) Let $d \geqslant 3$ and $\gamma \in\left[0, \frac{1}{6}\right]$. Then for each $n \in \mathbb{Z}^{d}$ the following estimate

$$
\int_{1}^{\infty} t^{\gamma}\left|\left(e^{ \pm i t \Delta}\right)(n)\right| d t \leqslant \beta
$$

holds true, where

$$
\beta=\sup _{m \in \mathbb{Z}} \int_{1}^{\infty} t^{\gamma}\left|J_{m}(t)\right|^{d} d t<\infty .
$$

(ii) Let a function $q \in \ell^{2}\left(\mathbb{Z}^{d}\right)$ and let $X(\lambda)=q R_{0}(\lambda) q, \lambda \in \Lambda$. Then the operatorvalued function $X: \Lambda \rightarrow \mathcal{B}_{2}$ is analytic and satisfies

$$
\begin{aligned}
& \sup _{\lambda \in \Lambda}\|X(\lambda)\|_{\mathcal{B}_{2}} \leqslant(1+\beta)\|q\|_{2}^{2}, \\
& \left\|X(\lambda)-X\left(\lambda_{1}\right)\right\|_{\mathcal{B}_{2}} \leqslant(1+\beta)\|q\|_{2}^{2}\left|\lambda-\lambda_{1}\right|^{\gamma}, \quad \forall \lambda, \lambda_{1} \in \Lambda .
\end{aligned}
$$

Proof (i) Note that (7.12) gives (7.15). Due to (7.2) it is sufficient to show (7.14) for $n \in\left(\mathbb{Z}_{+}\right)^{d}$. Using (7.11) and (7.15), we obtain

$$
\int_{1}^{\infty}\left|\left(e^{i t \Delta}\right)(n)\right| d t=\int_{1}^{\infty} \prod_{1}^{d}\left|J_{n_{j}}(t)\right| d t \leqslant \prod_{1}^{d}\left(\int_{1}^{\infty}\left|J_{n_{j}}(t)\right|^{d}\right)^{1 / d} \leqslant \beta
$$

which yields (7.14).

(ii) Consider the case $\mathbb{C}_{-}$, the proof for $\mathbb{C}_{+}$is similar. We have the standard representation of the free resolvent $R_{0}(\lambda)$ in the lower half-plane $\mathbb{C}_{-}$given by

$$
\begin{aligned}
& R_{0}(\lambda)=-i \int_{0}^{\infty} e^{i t(\Delta-\lambda)} d t=R_{01}(\lambda)+R_{02}(\lambda) \\
& R_{01}(\lambda)=-i \int_{0}^{1} e^{i t(\Delta-\lambda)} d t, \quad R_{02}(\lambda)=-i \int_{1}^{\infty} e^{i t(\Delta-\lambda)} d t
\end{aligned}
$$

for all $\lambda \in \mathbb{C}_{-}$. Here the operator valued-function $R_{01}(\lambda)$ has analytic extension from $\mathbb{C}_{-}$into the whole complex plane $\mathbb{C}$ and satisfies

$$
\left\|R_{01}(\lambda)\right\| \leqslant 1, \quad\left\|q R_{01}(\lambda) q\right\|_{\mathcal{B}_{2}} \leqslant\|q\|_{2}^{2} \forall \lambda \in \mathbb{C}_{-},
$$

Let $R_{02}\left(n^{\prime}-n, \lambda\right)$ be the kernel of the operator $R_{02}(\lambda)$. We have the identity

$$
R_{02}(m, \lambda)=-i \int_{1}^{\infty}\left(e^{i t(\Delta-\lambda)}\right)(m) d t, \quad m=n^{\prime}-n .
$$

Then the estimate (7.14) gives

$$
\left|R_{02}(m, \lambda)\right| \leqslant \int_{1}^{\infty}\left|\left(e^{i t \Delta}\right)(m)\right| d t \leqslant \beta
$$


which yields

$$
\begin{aligned}
\left\|q R_{02}(\lambda) q\right\|_{\mathcal{B}_{2}}^{2} & =\sum_{n, n^{\prime} \in \mathbb{Z}^{d}}|q(n)|^{2}\left|R_{02}\left(n-n^{\prime} \lambda\right)\right|^{2}|q(n)|^{2} \\
& \leqslant \sum_{n, n^{\prime} \in \mathbb{Z}^{d}}|q(n)|^{2} \beta^{2}|q(n)|^{2}=\beta^{2}\|q\|_{2}^{4}
\end{aligned}
$$

and summing results for $R_{01}$ and $R_{02}$ we obtain (7.16). The proof of (7.16) is similar.

Acknowledgements Various parts of this paper were written during Evgeny Korotyaev's stay in KTH and Mittag-Leffler Institute, Stockholm. He is grateful to the institutes for the hospitality. He is also grateful to Alexei Alexandrov (St. Petersburg) and Konstantin Dyakonov (Barcelona), Nikolay Shirokov (St. Petersburg) for stimulating discussions and useful comments about Hardy spaces. Our study was supported by the RSF Grant No. 15-11-30007.

Open Access This article is distributed under the terms of the Creative Commons Attribution 4.0 International License (http://creativecommons.org/licenses/by/4.0/), which permits unrestricted use, distribution, and reproduction in any medium, provided you give appropriate credit to the original author(s) and the source, provide a link to the Creative Commons license, and indicate if changes were made.

\section{References}

1. Abramov, A.A., Aslanyan, A., Davies, E.B.: Bounds on complex eigenvalues and resonances. J. Phys. A 34, 57-72 (2001)

2. Ando, K.: Inverse scattering theory for discrete Schrödinger operators on the hexagonal lattice. Ann. Henri Poincaré 14, 347-383 (2013)

3. Bateman, H., Erdelyi, A.: Higher Transcendental Functions, vol. 2. McGraw-Hill Book Company, New York (1953)

4. Borichev, A., Golinskii, L., Kupin, S.: A Blaschke-type condition and its application to complex Jacobi matrices. Bull. Lond. Math. Soc. 41, 117-123 (2009)

5. Boutet de Monvel, A., Sahbani, J.: On the spectral properties of discrete Schrödinger operators: (the multi-dimensional case). Rev. Math. Phys. 11, 1061-1078 (1999)

6. Davies, E.B.: Non-self-adjoint differential operators. Bull. Lond. Math. Soc. 34(5), 513-532 (2002)

7. Davies, E.B., Nath, J.: Schrödinger operators with slowly decaying potentials. J. Comput. Appl. Math. 148, 1-28 (2002)

8. Demuth, M., Hansmann, M., Katriel, G.: On the discrete spectrum of non-selfadjoint operators. J. Funct. Anal. 257(9), 2742-2759 (2009)

9. Demuth, M., Hansmann, M., Katriel, G.: Eigenvalues of non-selfadjoint operators: a comparison of two approaches. In: Demuth, M., Kirsch, W. (eds.) Mathematical Physics, Spectral Theory and Stochastic Analysis, pp. 107-163. Springer, Basel (2013)

10. Duren, P.L.: Theory of $H^{p}$ Spaces. Academic Press, San Diego (1970)

11. Frank, R.L.: Eigenvalue bounds for Schrödinger operators with complex potentials. Bull. Lond. Math. Soc. 43(4), 745-750 (2011)

12. Frank, R.L.: Eigenvalue bounds for Schrödinger operators with complex potentials. III. Trans. Am. Math. Soc. 370(1), 219-240 (2018)

13. Frank, R.L., Laptev, A., Lieb, E.H., Seiringer, R.: Lieb-Thirring inequalities for Schrödinger operators with complex-valued potentials. Lett. Math. Phys. 77, 309-316 (2006)

14. Frank, R.L., Laptev, A., Safronov, O.: On the number of eigenvalues of Schrödinger operators with complex potentials. J. Lond. Math. Soc. (2) 94(2), 377-390 (2016)

15. Frank, R.L., Sabin, J.: Restriction theorems for orthonormal functions, Strichartz inequalities and uniform Sobolev estimates. Am. J. Math. 139(6), 1649-1691 (2017) 
16. Frank, R.L., Laptev, A., Seiringer, R.: A sharp bound on eigenvalues of Schrödinger operators on the half-line with complex-valued potentials. Oper. Theory Adv. Appl. 214, 39-44 (2011)

17. Frank, R.L., Simon, B.: Eigenvalue bounds for Schrödinger operators with complex potentials. II. J. Spectr. Theory 7(3), 633-658 (2017)

18. Garnett, J.: Bounded Analytic Functions. Academic Press, New York (1981)

19. Gohberg, I.C., Krein, M.G.: Introduction to the Theory of Linear Non-selfadjoint Operators in Hilbert Space, Translation of Mathematical Monographs, 18. AMS, Providence (1969)

20. Hoffman, K.: Banach Spaces of Analytic Functions. Prentice-Hall, Englewood Cliffs (1962)

21. Isozaki, H., Korotyaev, E.: Inverse problems, trace formulae for discrete Schrödinger operators. Ann. Henri Poincare 13(4), 751-788 (2012)

22. Isozaki, H., Morioka, H.: A Rellich type theorem for discrete Schrödinger operators. Inverse Probl. Imaging 8(2), 475-489 (2014)

23. Koosis, P.: Introduction to $H_{p}$ Spaces. Cambridge Tracts in Mathematics, vol. 115. Cambridge University Press, Cambridge (1998)

24. Kopylova, E.A.: Dispersive estimates for discrete Schrödinger and Klein-Gordon equations. St. Petersbg. Math. J. 21(5), 743-760 (2010)

25. Korotyaev, E., Saburova, N.: Scattering on periodic metric graphs. arXiv:1507.06441

26. Laptev, A., Safronov, O.: Eigenvalue estimates for Schrödinger operators with complex potentials. Commun. Math. Phys. 292, 29-54 (2009)

27. Lieb, E.H., Thirring, W.: Inequalities for the Moments of the Eigenvalues of the Schrödinger Hamiltonian and Their Relation to Sobolev Inequalities. Studies in Mathematical Physics, pp. 269-303. Princeton University Press, Princeton (1976)

28. Lieb, E., Loss, M.: Analysis. Graduate Studies in Mathematics, vol. 14. AMS, Providence (1997)

29. Olver, F.W.J.: Asymptotics and Special Functions. Academic Press, New York (1974)

30. Rosenblum, G., Solomjak, M.: On the spectral estimates for the Schrödinger operator on $\mathbb{Z}^{d}, d \geqslant 3$. J. Math. Sci. N. Y. 159(2), 241-263 (2009)

31. Reed, M., Simon, M.B.: Methods of Modern Mathematical Physics. II. Fourier Analysis, SelfAdjointness. Academic Press, New York (1975)

32. Reed, M., Simon, B.: Methods of Modern Mathematical Physics, IV: Analysis of Operators. Academic Press, New York (1978)

33. Safronov, O.: Estimates for eigenvalues of the Schrödinger operator with a complex potential. Bull. Lond. Math. Soc. 42(3), 452-456 (2010)

34. Safronov, O.: On a sum rule for Schrödinger operators with complex potentials. Proc. Am. Math. Soc. 138(6), 2107-2112 (2010)

35. Shaban, W., Vainberg, B.: Radiation conditions for the difference Schrödinger operators. J. Appl. Anal. 80, 525-556 (2001)

36. Smirnov, V.I.: A Course of Higher Mathematics, vol. 3. Part 2: Complex Variables Special Functions. (International Series of Monographs in Pure and Applied Mathematics; Volume 60). Pergamon Press, New York (1964)

37. Toda, M.: Theory of Nonlinear Lattices, 2nd edn. Springer, Berlin (1989)

Publisher's Note Springer Nature remains neutral with regard to jurisdictional claims in published maps and institutional affiliations. 